\title{
The de novo genome assembly of Tapiscia sinensis and the transcriptomic and developmental bases of androdioecy
}

\author{
Peng Zhao', Guiliang Xin ${ }^{1}$, Feng Yan' ${ }^{1}$, Huan Wang ${ }^{1}$, Xiaolong Ren', Keith Woeste $\mathbb{B}^{2}$ and Wenzhe Liu ${ }^{1}$
}

\begin{abstract}
Tapiscia sinensis (Tapisciaceae) possesses an unusual androdioecious breeding system that has attracted considerable interest from evolutionary biologists. Key aspects of $T$. sinensis biology, including its biogeography, genomics, and sexlinked genes, are unknown. Here, we report the first de novo assembly of the genome of $T$. sinensis. The genome size was $410 \mathrm{Mb}$, with 22,251 predicted genes. Based on whole-genome resequencing of 55 trees from 10 locations, an analysis of population genetic structure indicated that $T$. sinensis has fragmented into five lineages, with low intrapopulation genetic diversity and little gene flow among populations. By comparing whole-genome scans of male versus hermaphroditic pools, we identified 303 candidate sex-linked genes, 79 of which (25.9\%) were located on scaffold 25. A 24-kb region was absent in hermaphroditic individuals, and five genes in that region, TsF-box4, TsF-box10, TSF-box13, TSSUT1, and TSSUT4, showed expression differences between mature male and hermaphroditic flowers. The results of this study shed light on the breeding system evolution and conservation genetics of the Tapisciaceae.
\end{abstract}

\section{Introduction}

Tapiscia sinensis Oliv. (Tapisciaceae), is a woody, perennial, androdioecious species ${ }^{1-6}$. Androdioecy is a rare breeding system in which populations consist of only male and hermaphroditic individuals ${ }^{7,8}$. Hermaphroditic individuals have both male and female functions ${ }^{9}$. Functional androdioecy is found in less than $0.005 \%$ of angiosperms $^{10}$, including Mercurialis annua ${ }^{11}$, Datisca glomerata $^{11,12}$, Schizopepon bryoniaefolius ${ }^{9}$, Laguncularia racemosa ${ }^{13}$, and Osmanthus fragrans ${ }^{14,15}$. T. sinensis has research value as a model for the study of the evolution and maintenance of androdioecy ${ }^{1,2,4}$. In T. sinensis, floral buds on hermaphroditic individuals initiate differentiation in May, flower in late June, and are fertilized in early

\footnotetext{
Correspondence: Wenzhe Liu (Iwenzhe@nwu.edu.cn)

${ }^{1}$ Key Laboratory of Resource Biology and Biotechnology in Western China, Ministry of Education, College of Life Sciences, Northwest University, Xi'an,

Shaanxi 710069, China

${ }^{2}$ USDA Forest Service Hardwood Tree Improvement and Regeneration Center (HTIRC), Department of Forestry and Natural Resources, Purdue University,

715 West State Street, West Lafayette, IN 47907, USA

These authors contributed equally: Peng Zhao, Guiliang Xin
}

July ${ }^{1,2}$. After 9 months of quiescence, young fruits resume development in April, reaching maturity in September. Thus, the flower and fruit development of $T$. sinensis lasts 17 months ${ }^{1}$, a phenomenon more common in gymnosperms ${ }^{16,17}$. Other angiosperms with long flower and fruit development $^{18,19}$ include Carpinus turczaninowii, Ostryopsis davidiana ${ }^{18}$, Betula platyphylla ${ }^{19}$, and trees in section Lobatae of Quercus.

Tapiscia was formerly widespread in the Northern Hemisphere. More than ten fossil species are described among the Eocene flora ( 60 million years ago, Ma) of China $^{20}$, England ${ }^{21}$, Germany ${ }^{21}$, Oregon (USA) ${ }^{22}$, and Canada $^{23}$ (Fig. 1a). Currently, the genus comprises a single species, T. sinensis, which is distributed in central, subtropical mountains of China, south of the Yangtze River between elevations of 250 and $2200 \mathrm{~m}$, and in northern Vietnam ${ }^{1-5,24,25}$. Due to deforestation and reclamation, natural populations of $T$. sinensis are rare [International Union for Conservation of Nature (IUCN) Red List]; it grows only in small, scattered, disjunct sites, mostly at higher elevations ${ }^{25-28}$. The phylogenetic

\section{(c) The Author(s) 2020}

(c) (i) Open Access This article is licensed under a Creative Commons Attribution 4.0 International License, which permits use, sharing, adaptation, distribution and reproduction cc) in any medium or format, as long as you give appropriate credit to the original author(s) and the source, provide a link to the Creative Commons license, and indicate if changes were made. The images or other third party material in this article are included in the article's Creative Commons license, unless indicated otherwise in a credit line to the material. If material is not included in the article's Creative Commons license and your intended use is not permitted by statutory regulation or exceeds the permitted use, you will need to obtain permission directly from the copyright holder. To view a copy of this license, visit http://creativecommons.org/licenses/by/4.0/. 

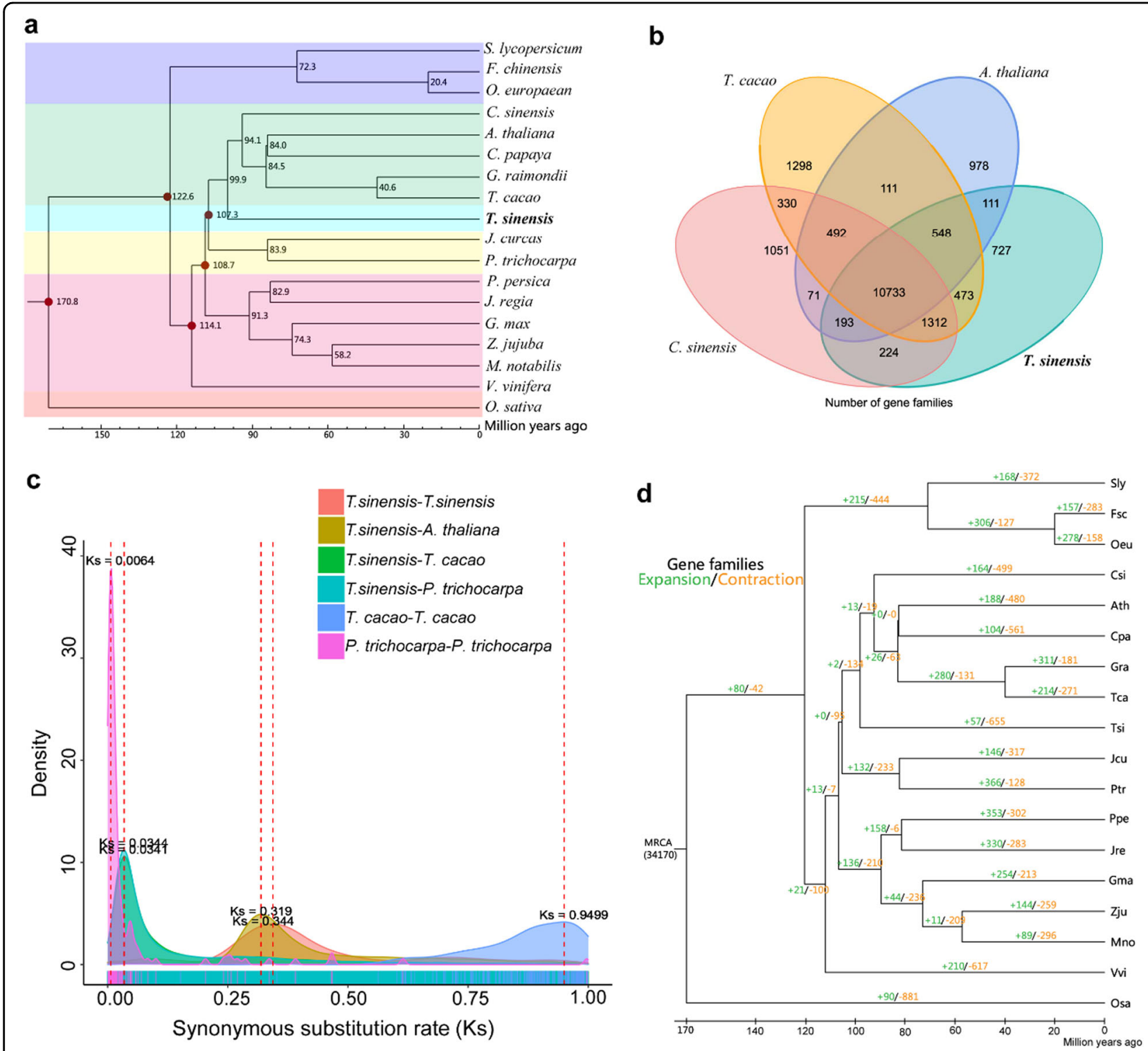

Fig. 1 Comparative analysis of the Tapiscia sinensis genome with the genomes of other plant species. a Phylogenetic analysis of $T$. sinensis and other sequenced plants. O. sativa was used as an outgroup. $\mathbf{b}$ Venn diagram of shared orthologous gene families in $T$. sinensis, A. thaliana, T. cacao, and C. sinensis. c Density distributions of the synonymous substitution rate (Ks) between duplicated genes of syntenic regions in $A$. thaliana, P. trichocarpa, T. cacao, and T. sinensis are shown by different colors. $\mathbf{d}$ Phylogenetic tree and expansion and contraction of gene families. The phylogenetic tree was constructed from a concatenated alignment of 509 families of single-copy genes from 18 higher plant species. Sly, S. Iycopersicum; Fsc, F. chinensis; Oue, O. europaea; Csi C. sinensis; Ath, A. thaliana; Cpa, C. papaya; Gra, G. raimondii; Tca, T. cacao; Tsi, T. sinensis; Jcu, J. curcas; Ptr, P. trichocarpa; Gma, G. max; Jre, J. regia; Ppe, P. persica; Zju, Z. jujube; Mno, M. notabilis; Vvi, V. vinifera; Osa, O._sativa (details in Supplementary Table 11). MRCA is the most recent common ancestor. Gene family expansions are indicated in green, and gene family contractions are indicated in orange

position of $T$. sinensis makes it an important species for understanding angiosperm evolution. In addition, this tree species has great value in Chinese traditional medicine and in landscape horticulture $e^{2,25}$.

Here, we report the sequencing and de novo assembly of the genome of a $T$. sinensis individual growing in the Qinling Mountains, Shaanxi Province. We used the annotated genome to determine the number of proteincoding genes in Tapiscia; to evaluate its genome structure, including whole-genome duplication; to evaluate its phylogenetic position; and to estimate the timing of its divergence from its most recent ancestor. An important objective of this research was to identify regions of the Tapiscia genome linked to sex determination and to 
identify and describe the expression of genes that contribute to Tapiscia's unusual reproductive habit.

\section{Results}

Genome sequencing, assembly, repeat sequence annotation, and gene prediction

We sequenced a single genotype of $T$. sinensis; the source tree was a wild male individual from the Qinling Mountains, China. The input data for the whole-genome assembly included three libraries with insert sizes from $350 \mathrm{bp}$ to $20 \mathrm{~kb}$ (Supplementary Table 1). Most of the data were sequences from the ends of short $(400 \mathrm{bp})$ fragments; $59.30 \mathrm{~Gb}$ were sequenced from an Illumina pairedend library with an insert size of $350 \mathrm{bp}$. The sequence data represented $\sim 131.97 \times$ coverage of the genome (Supplementary Table 1). We generated longer-fragment libraries of $20 \mathrm{~kb}$ that assisted greatly in linking contigs into scaffolds using the PacBio Sequel platform. Using the PacBio platform, we generated $39.96 \mathrm{~Gb}$ of sequence data representing approximately $88.93 \times$ coverage of the genome. We also sequenced $54.93 \mathrm{~Gb}$ of data using the $10 \mathrm{X}$ Genomics platform, which generates $500-700 \mathrm{bp}$ read lengths. These data represented $\sim 122.25 \times$ coverage of the genome. The final genome assembly was $410 \mathrm{Mb}$, with contig and scaffold N50 sizes of 1.72 and $4.64 \mathrm{~kb}$, respectively (Table 1 and Supplementary Tables 2, 3). The

Table 1 Statistics and BUSCO evaluation of the Tapiscia sinensis genome

\begin{tabular}{ll}
\hline Estimate of genome size & $\mathbf{4 1 0} \mathbf{~ M b}$ \\
\hline GC content & $33.41 \%$ \\
N50 length (contig) & $1757 \mathrm{bp}$ \\
Number of contigs & 836,436 \\
N50 length (scaffold) & $4747 \mathrm{bp}$ \\
Number of scaffolds & 557 \\
Number of genes & 22,251 \\
Average gene length & $4990 \mathrm{bp}$ \\
Average coding sequence length & $1210 \mathrm{bp}$ \\
Average exon length & $302 \mathrm{bp}$ \\
Average TE protein length & $278 \mathrm{bp}$ \\
Tandem repeat & $228,544 \mathrm{bp}$ \\
Complete BUSCOs & 2267 (97.5\%) \\
Complete and single-copy BUSCOs & 2153 (92.6\%) \\
Complete and duplicated BUSCOs & $113(4.9 \%)$ \\
Fragmented BUSCOs & 20 (0.9\%) \\
Missing BUSCOs & $37(1.6 \%)$ \\
Total BUSCO groups searched & 2326 (100\%) \\
\hline
\end{tabular}

final assembly size was $\sim 96 \%$ of the estimated total genome size based on 17-mer analysis $(427.77 \mathrm{Mb})$ (Supplementary Figs. 1, 2). A total of $154.19 \mathrm{~Gb}$ of sequence resulted in $\sim 343.15 \times$ coverage of the genome. We identified 2267 complete benchmarking universal single-copy orthologs (BUSCOs), including 2153 single-copy BUSCOs, 113 duplicated BUSCOs, 20 fragmented BUSCOs, and 37 missing BUSCOs, in the assembled Tapiscia sinensis genome (Table 1). The chloroplast genome of $T$. sinensis was assembled separately into one circular genome with a size of $161,100 \mathrm{bp}$, including a large singlecopy (LSC) region of 87,766 bp, a small single-copy (SSC) region of $18,520 \mathrm{bp}$, and an inverted repeat region (IRa and IRb) of $\sim 27,407 \mathrm{bp}^{27}$. The tandem repeat (long terminal repeat, LTR) sequence annotations showed that transposable elements accounted for $\sim 5.2 \%$ of the reference genome (Table 1 and Supplementary Table 2). The GC content was $\sim 33.41 \%$ across the T. sinensis genome.

\section{Transcriptome sequencing and assembly}

We generated a total of $29.7 \mathrm{~Gb}$ of RNA sequence data from male flowers, hermaphroditic flowers, fruits, roots, bark, leaves, young stems (tender shoots), and mature stems to annotate the genome (Supplementary Table 4). Trinity splicing results displayed 147,609 subclusters in PASA software by alignment clustering. The transcriptome sequences covered $84.91 \%$ of the reference genome. Over 456 million paired-end reads were sequenced across the six tissues. As a measure of the quality of the genome assembly of T. sinensis, $77.34 \%$ of 197,283 unigenes from the RNA-seq data were aligned to the genome (Supplementary Table 5). More than $80 \%$ of transcript reads could be mapped to the Illumina reads, with only slight variation based on tissue source [fruit $(88.51 \%)$, root $(88.10 \%)$, bark $(88.42 \%)$, leaf $(80.52 \%)$, young stem $(90.50 \%)$, and mature stem $(88.07 \%)]$ (Supplementary Tables 4, 5). We defined 22,251 proteincoding genes (Table 1 and Supplementary Tables 2, 3, 6). Homology-based gene searches and searches for predicted noncoding RNA (ncRNA) (Supplementary Table 2) yielded 1180 ribosomal RNA (rRNA) genes, 497 transfer RNA (tRNA) genes, 383 small nuclear RNA (snRNA) genes, and 364 microRNA (miRNA) genes (Supplementary Table 2).

A total of 92,073 simple sequence repeats (SSRs) were found in T. sinensis (Supplementary Tables 7-9). We annotated 72,706 SSRs for studying the population genetics of T. sinensis (Supplementary Tables 7-9). We designed 150 pairs of primers and found 68 polymorphic loci (45.5\%). Six polymorphic SSRs were used for genetic analysis of $51 \mathrm{~T}$. sinensis individuals ${ }^{28}$. We used the sexlinked SSR locus TS095 (forward: TTGTCCCTCT CAACTTCGCT, reverse: AAAATCAACCAGCCAGTT CG) ${ }^{28}$ to evaluate $1041 \mathrm{~T}$. sinensis offspring (seeds) from 
five locations, including the Northwest University campus, Qinling Mountains, Wuling Mountains, Daba Mountains, and Luoxiao Mountains (Supplementary Table 10). The results based on segregation of the TS095 SSR alleles showed that the sex ratio among these 1041 offspring was nearly $1: 1$; the numbers of males and hermaphrodites were 505 and 536, respectively (Supplementary Table 10).

\section{Comparative analysis of the $T$. sinensis genome and gene-based phylogeny}

The annotated gene models $(N=22,251)$ were grouped into gene families $(N=6,767)$ comprising at least two genes based on sequence similarity. We identified $229 T$. sinensis-specific genes that did not cluster with any genes from any of 17 other plant species: Morus notabilis, Ziziphus jujuba (jujube), Prunus persica (peach), Oryza sativa (rice), Arabidopsis thaliana, Juglans regia (common walnut), Vitis vinifera (grape), Jatropha curcas, Glycine max (soybean), Populus trichocarpa (poplar), Gossypium raimondii (cotton), Theobroma cacao (cocoa), Fraxinus chinensis, Carica papaya (papaya), Solanum lycopersicum (tomato), Olea europaea and Citrus sinensis (orange) (Supplementary Table 11). A total of 10,733 gene families were found based on comparisons among $T$. sinensis, $C$. sinensis, A. thaliana, and T. cacao; this four-species comparison revealed 727 species-specific genes in $T$. sinensis (Fig. 1b and Supplementary Tables 12, 13). We constructed a phylogenetic tree of $T$. sinensis together with 17 other sequenced plant genomes based on 594 single-copy genes using the monocot rice (O. sativa) as an outgroup (Fig. 1a and Supplementary Table 1 and Supplementary Fig. 3). The resulting tree shows that $T$. sinensis diverged approximately $100 \mathrm{Ma}$ from a clade containing the Rutaceae, Brassicaceae, and Malvaceae, among others (Fig. 1a).

The distribution of Ks values peaked at approximately 0.34 in $T$. sinensis, indicating that $T$. sinensis has not undergone any recent lineage-specific whole-genome duplication (WGD) events (Fig. 1c). The distribution of $4 \mathrm{DTv}$ values at approximately 0.45 supported this interpretation of the fourfold degenerate site (4DTv) results (Supplementary Fig. 4). Pairwise orthology between species, including the orthology between $T$. sinensis and $P$. trichocarpa (Salicaceae), T. sinensis and T. cacao, and T. sinensis and $A$. thaliana, showed $4 \mathrm{DTv}$ distance peaks at $\sim 0.30, \sim 0.30$, and 0.60 , respectively, indicating that the divergence time of $T$. sinensis from $A$. thaliana was earlier than that from $P$. trichocarpa, consistent with the phylogenetic tree (Fig. 1a).

To identify gene families that had expanded or contracted only in $T$. sinensis, we compared gene families from $T$. sinensis with those of 17 other representative species and with an ancestral species. We identified a total of 57 gene families that have undergone significant $(p<$ 0.01 ) expansion in the T. sinensis genome (Supplementary Table 14). The results of Kyoto Encyclopedia of Genes and Genomes (KEGG) pathway enrichment analysis revealed that these families were enriched with genes involved in phototransduction, anthocyanin biosynthesis, calcium signaling, oocyte meiosis, and plant-pathogen interaction, indicating that these pathways have evolved distinctly in $T$. sinensis compared to other plant species. Information about enrichment provides a basic resource for understanding secondary metabolism in $T$. sinensis (Supplementary Tables 14-16). Analysis of gene ontology (GO) and KEGG pathways showed that male and hermaphroditic plants were enriched in different annotations; for example, in males, we found that the greatest number of enriched genes were related to metabolic process, catalytic activity, and hydrolase activity, whereas enrichment in the hermaphroditic plant genome was in binding genes (Fig. 4b and Supplementary Figs. 5, 6 and Supplementary Table 17).

\section{Genetic diversity and population structure of $T$. sinensis}

To investigate the genomic diversity, population structure, and biogeography of $T$. sinensis, we sequenced 55 trees from 10 locations that represent the species' entire natural distribution in China (Fig. 2a, b and Supplementary Table 18). Thus, we generated a total of $560 \mathrm{~Gb}$ of high-quality, cleaned sequence data, at an average of $10.19 \mathrm{~Gb}$ per sample (equivalent to $\sim 25 \times$ coverage of the $\sim 410-\mathrm{Mb}$ sequence of the wild male individual "Tree168"). Sequences of Tree 168 were also mapped to the reference genome (mapping rate of 90.04\%) (Supplementary Table 19). A total of 11,431,841 single nucleotide polymorphisms (SNPs) were identified among 55 individuals, with an average of $\sim 27$ SNPs per $\mathrm{kb}$ of the $T$. sinensis genome (Supplementary Table 19). A total of $62.1 \%$ of these SNPs were intergenic; $4.2 \%$ of the SNPs were located in coding sequences (CDS), $2.12 \%$ were synonymous, and $2.06 \%$ were nonsynonymous, resulting in a nonsynonymous/synonymous ratio of 0.97 (Supplementary Table 20).

We analyzed the population structure of $55 \mathrm{~T}$. sinensis trees using three methods: STRUCTURE runs on genomic SNPs, a neighbor-joining (NJ) tree, and a principal component analysis (PCA) of all SNPs (Fig. 2b-d and Supplementary Fig. 7). The clearest differentiation was found in the nuclear genome; five distinct groups emerged from the analysis. The populations of Zhejiang Province and the Tianmu Mountains (TM and BM, respectively, yellow in Fig. 2b) were the most geographically isolated and most genetically distinct (Fig. 2). The Yunnan Province $(\mathrm{YN})$ population (southwestern China) was the second most genetically distinct. The third group was centered in the Qinling Mountains (NX and ND), the 


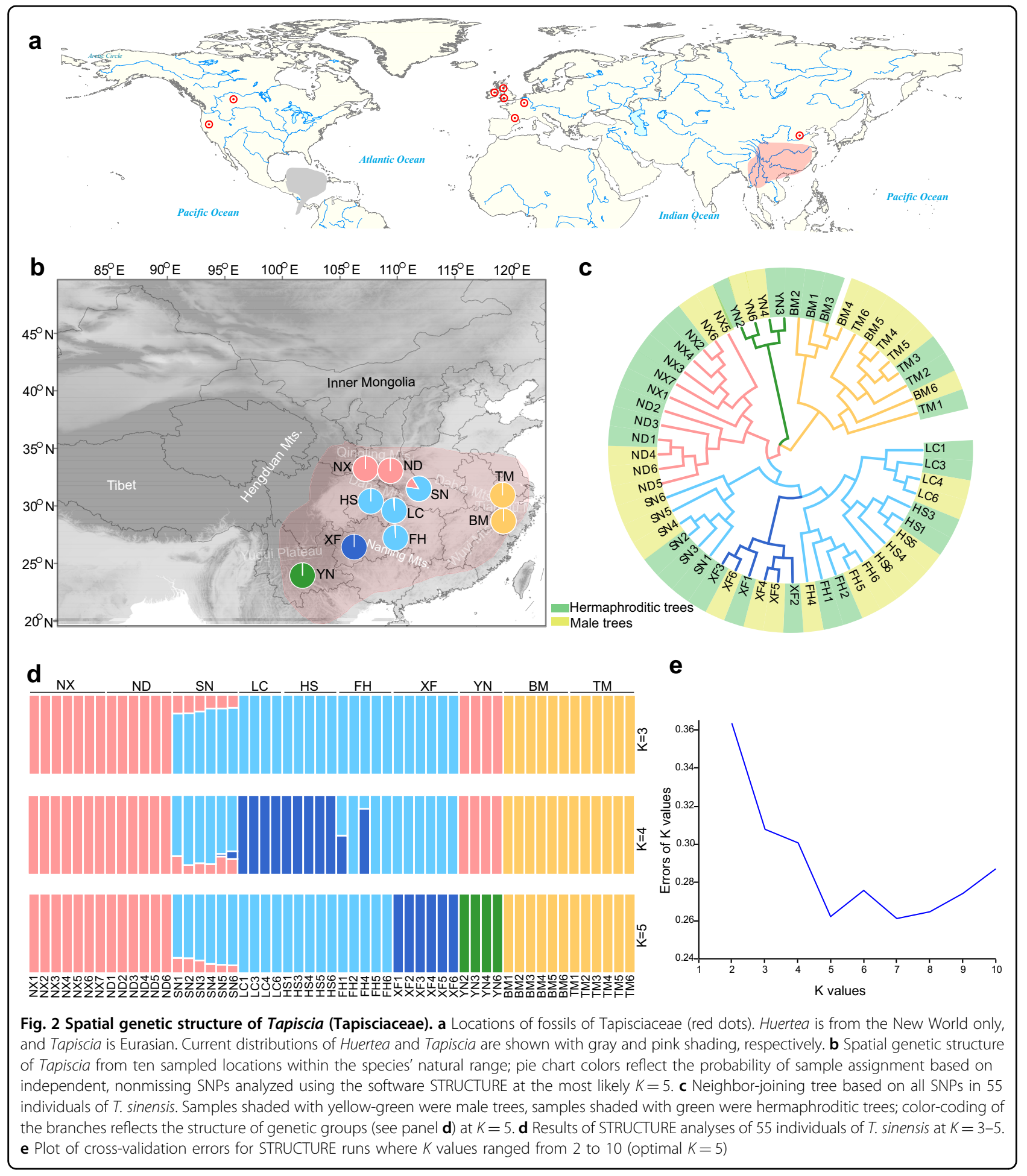

fourth population was from Guizhou Province (XF), and the fifth group of samples constituted a population in Hubei Province [(Shennongiia national forest (SN) and Lichuan County (LC)], Hunan Province (FH), and Chongqing (HS) (Fig. 2 and Supplementary Fig. 7 and Supplementary Table 18).
Bayesian analysis of the genetic structure of the 55 trees from all 10 sampled sites produced populations that corresponded exactly with the clusters identified using neighbor joining (Fig. 2b-d and Supplementary Fig. 7). The underlying hierarchical structure of the $T$. sinensis trees was best represented at $K=5$, in part because the 
individuals clustered into populations that corresponded to the geographic regions from which they were sampled (Fig. 2e). The results of PCA revealed five clusters of samples corresponding to the same groups identified by STRUCTURE and further supported the genetic distinctiveness of populations TM and BM (Fig. 2 and Supplementary Fig. 7). The distribution of T. sinensis in China is marked by strong fragmentation. The populations of Zhejiang, Yunnan, and Guizhou are highly differentiated genetically from those of other regions, and there was no gene flow signal into these populations from populations in other regions. Two populations of T. sinensis growing in the Qinling Mountains clustered into one genetic component. T. sinensis trees in the Shennongjia Mountains $(\mathrm{SN})$ population showed evidence of gene introgression from the NX and ND populations (Fig. 2).

\section{Morphology and development of male and hermaphroditic flowers}

We compared the morphological differentiation of male versus hermaphroditic flowers (Fig. 3). T. sinensis flowers consist of five stamens with greenish filaments and yellow anthers $1-2 \mathrm{~mm}$ in length. In hermaphroditic flowers, the ovary is unilocular with 1 ovule; the style is longer than the stamens in bisexual flowers (Fig. 3d, e). In male flowers, the ovary is present but vestigial (Fig. 3d, e). Scanning electron microscope (SEM) images showed that there were no obvious differences between male flowers (MFs) and hermaphroditic flowers (HFs) before stage 5 (Fig. 3H, h). At stage 6, however, in hermaphroditic flowers, a ring meristem forms as a ridge around the central zone of the flower (Fig. 3I, J). At stage 7, the gynoecium grows as a hollow tube (Fig. 3J); from stages 11 to 14 (during flowering), the gynoecium becomes ready for fertilization, and when the stamens extend out from the petals, fertilization occurs (Fig. 3K, L). In contrast, at stage 6, in male flowers, the primordium of the gynoecium begins to form as a small bulge (Fig. 3i, j), unlike the apex of bisexual flowers (Fig. 3I). Male flowers produce a solid, nonfunctional, pistil-like structure (Fig. 3e, k). Bisexual flowers shed spherical pollen grains with a perforate tectum (Fig. 3F, G), while male flowers produced truncate pollen grains with a reticulate tectum (Fig. 3f, g).

\section{Selection associated with the sex-linked genomic regions of $T$. sinensis}

To identify sex-linked genomic regions of $T$. sinensis, we compared the genomes of 26 hermaphroditic individuals and 29 male individuals sampled from a variety of populations (Supplementary Table 18). Using $F_{\mathrm{ST}}$ values (cutoff set to 10E-5), we identified 303 candidate sexlinked genes (Fig. 4a, b and Supplementary Table 21). A high proportion $(N=79,26.1 \%)$ of these candidates were located on scaffold 25 (Fig. 4c and Supplementary Table
21). The genes in males with the greatest $F_{\mathrm{ST}}$ were involved in amine metabolism, catalytic activity, and hydrolase activity. In hermaphroditic plants, the genes with the greatest $F_{\mathrm{ST}}$ were related to RNA binding and calcium ion binding. Other genes on scaffold 25 of hermaphroditic plants included phospholamban $(P L N)$, late embryogenesis abundant ( $L E A)$, glyoxalase $(G l y)$, major facilitator superfamily (MFS), ribosomal-S25 (RPL), $50 \mathrm{~S}$ ribosome-binding GTPase $(R b g A)$, ferredoxin $(F d x)$, and ribosomal protein 1 10A (RPL10A) (Fig. 4c and Supplementary Table 21). We chose to analyze these loci further for insights into the genomic basis of androdioecy in $T$. sinensis. Among the 303 candidate sex-linked genes, we found that 64, 157, and 77 genes were expressed at significantly different levels in male flowers, hermaphroditic flowers, and fruits, respectively (Supplementary Fig. 8 and Supplementary Table 21). We further found that 34 of 303 genes $(11.2 \%)$ had $F_{\mathrm{ST}}$ values $>0.2$, nine of which were highly expressed in male flowers, seventeen of which were highly expressed in hermaphroditic flowers, and eight of which were highly expressed in fruits (Supplementary Fig. 8 and Supplementary Table 21).

A total of 13 TsRPL1OA genes were identified in the genome of $T$. sinensis using a search based on the conserved domain of A. thaliana RPL10A. The TsRPL10As were unevenly distributed among 11 scaffolds, but only TsRPL10A9 was located on scaffold 25 of T. sinensis. We analyzed the conserved domains and gene structure of the ribosomal_L1 superfamily RPL10A gene in male and hermaphroditic plants of $T$. sinensis and compared them to those in A. thaliana (Fig. 4d, e). The results showed that the TsRPL10A9 gene of male T. sinensis was similar to the A. thaliana RPL10A gene; by contrast, several large deletions were present in the same locus of hermaphroditic plants of $T$. sinensis (Fig. $4 \mathrm{~d}$, e and Supplementary Figs. 9-12 and Supplementary Table 22). We verified the sequences of TsRPL10A9 (male) and TsRPL10A9 (hermaphroditic) by Sanger sequencing (for details of the six primer pairs used to span the gene, see Supplementary Table 22 and Supplementary Figs. 9-12). We performed a sequence alignment of TsRPL10A9 (male) and TsRPL10A9 (hermaphrodite) with the reference genome and annotated the resulting combination with gene structure analysis (Fig. 4d-g and Supplementary Figs. 912). The first $2660 \mathrm{bp}$ of TsRPL10A9 were identical between male and hermaphroditic plants, and all seven coding sequences (CDS1 to CDS7) of TsRPL10A9 were present in male trees. In contrast, there were three deletions in exons of hermaphrodites that totaled $2410 \mathrm{bp}$ [978 bp, $659 \mathrm{bp}$, and $773 \mathrm{bp}$ located at CDS5 (5,423,343-5,423,399), CDS6 $(5,424,898-5,424,977)$, and CDS7 $(5,425,067-5,426,207)$, respectively]. These deletions in hermaphroditic plants were the major sources of sequence variation in TsRPL1OA genes between male and 


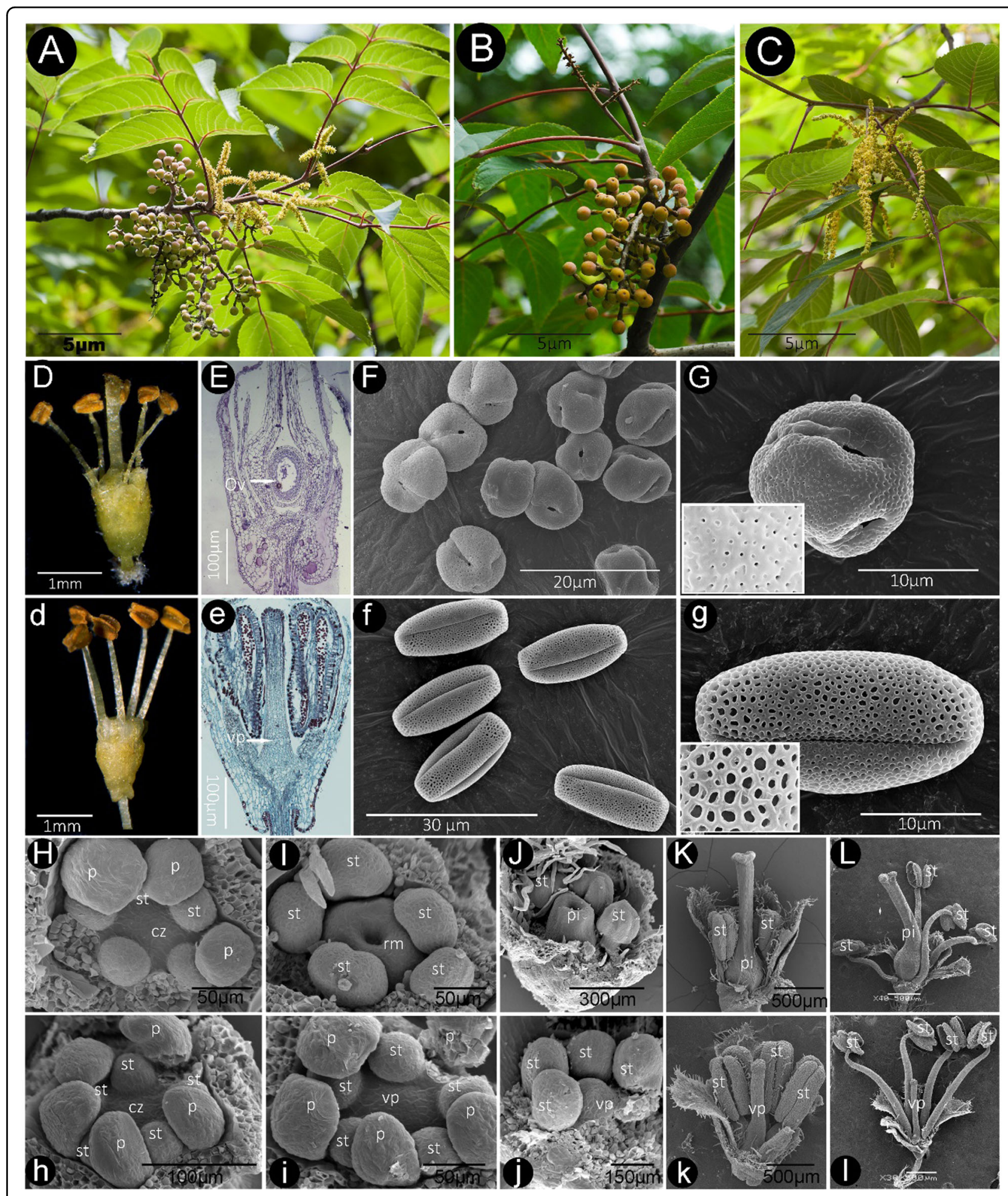

Fig. 3 (See legend on next page.) 
(see figure on previous page)

Fig. 3 Floral morphology and development of Tapiscia sinensis ${ }^{5,6}$. A Hermaphroditic T. sinensis individual showing the simultaneous occurrence of flowers and fruit. B Hermaphroditic T. sinensis individual with fruits of the previous year and fruits of the current year developing on the same branch. C Male inflorescence on a male individual. D-G Hermaphroditic flowers; D one pistil shedding pollen and five anthers; E longitudinal section of a hermaphroditic flower showing the pistil and unilocular ovary; $\mathbf{F}$ spherical pollen; $\mathbf{G}$ the exine sculpture of pollen grains, showing details of the perforate tectum. $\mathbf{d}-\mathbf{g}$ Male flower; $\mathbf{d}$ infertile pistil and five anthers; e longitudinal section of a male flower with no functional gynoecium; $\mathbf{f}$ olivary pollen; $\mathbf{g}$ the exine sculpture of pollen grains showing details of the reticulate tectum. $\mathbf{H}-\mathbf{L}, \mathbf{h}-\mathbf{I}$ Scanning electron micrographs of flowers; hermaphrodite $(\mathbf{H}-\mathbf{L})$ and male $(\mathbf{h}-\mathbf{I})$ at various developmental stages; carpel primordium phase of hermaphroditic $(\mathbf{H})$ and male $(\mathbf{h})$ flowers. Gynoecial ridge stage of hermaphroditic $(\mathbf{I}, \mathbf{J})$ and male $(\mathbf{i}, \mathbf{j})$ flowers (note: we did not observe a gynoecial ridge stage of male pistil development; five petals were removed to make the gynoecium visible). Fully differentiated but immature hermaphroditic $(\mathbf{K})$ and male (k) flowers. Mature hermaphroditic (L) and male (I) flowers. cz, Central zone; Ov, ovary; p, petal; pi, pistil; rm, ring meristem; st, stamen; vp, vestigial pistil

hermaphroditic plants (Fig. $4 \mathrm{f}-\mathrm{h}$ and Supplementary Figs. 9-12).

We examined the expression of TsRPL10As in $T$. sinensis tissues, including male and hermaphroditic flowers (Fig. 4h and Supplementary Tables 23, 24). Interestingly, most TsRPL10A genes were highly expressed in $T$. sinensis tissues, including fruits, male flowers, and hermaphroditic flowers (Fig. 4h and Supplementary Fig. 13), but two RPL10A genes (TsRPL10A4 and especially TSRPL10A9) showed higher expression in male and hermaphroditic flowers than in other tissues (Fig. 4h). Moreover, TsRPL10A9 was expressed at higher levels in male flowers than in hermaphroditic flowers (Fig. 4k).

F-box and sucrose transporter (SUT) family genes had high $F_{\mathrm{ST}}$ values, and members of these gene families were also on scaffold 25. For that reason, we investigated the transcript expression patterns of F-box and SUT family genes in T. sinensis tissues (Fig. 4i, $\mathbf{j}$ and Supplementary Fig. 14 and Supplementary Tables 23, 24). We found that three F-box genes (TsF-box4, TsF-box10, and TsF-box13) and two TsSUT genes (TsSUT1 and TsSUT4) were differentially expressed in male versus hermaphroditic flowers of T. sinensis (Fig. 4j and Supplementary Fig. 14 and Supplementary Tables 23, 24). Nine TsF-box genes (TsF-box1, TsF-box2, TsF-box5, TsF-box7, TsF-box9, TsFbox13, TsF-box14, TsF-box19, and TsF-box22) contained a conserved self-incompatibility (S-locus) domain (Supplementary Fig. 15), although self-incompatibility has not been investigated in $T$. sinensis. We found that the expression of TsRPL10A2, TsRPL10A7, TsRPL10A11, TsF-box14, and TsSUT4 was low in hermaphroditic individuals at stage 6 (Supplementary Figs. 13-15).

\section{Fruit quiescence in $T$. sinensis}

The bisexual flowers of $T$. sinensis bloom in June, but $T$. sinensis takes 17 months to complete the cycle from floral primordium initiation (in March or April) to fruit maturation. After fertilization, the young fruit develops rapidly. The receptacle becomes fleshy and swollen, wrapping the ovary and forming a gourd-shaped structure. Young fruits become quiescent in August; from September to February, fruits are in deep quiescence
(Fig. 5a), and their morphology and size remain unchanged until mid-April of the following year. In late March, flower development is initiated, and fruits reinitiate development; thus, flowers and fruits grow synchronously on hermaphroditic individuals (Fig. 3a). By the end of June, the current season's fruits and previous year's fruits are found on the same branch (Fig. 3b). Two months later (mid-August), the fruits of the preceding year are fully mature, and young fruits begin their quiescent period.

At the time of flowering, the ovary wall is composed of an inner and outer epidermis and 6-7 layers of parenchyma cells (Fig. 5b). In July, the parenchyma cells under the epidermis of the ovary wall develop a cork cambium that undergoes periclinal division to form phellem and phelloderm (Fig. 5b). In August, the phellogen cells in the ovary wall undergo periclinal division to form 3-6 layers of cork cells (outward) and one layer of phelloderm cells (inward) (Fig. 5b). The parts of the fruit protected by the remnant of the perianth and receptacle ("covered") do not form periderm (Fig. 5b). Abundant mucilage locules form in the naked pericarp and, at the same time, in the whole receptacle (Fig. 5b).

The molecular mechanisms underlying the regulation of $T$. sinensis fruit quiescence and embryo development are likely to be complex and strongly temporally regulated. To understand the regulation of the development of $T$. sinensis fruit, we integrated genomic and transcriptomic analyses of genes in T. sinensis fruit at eight stages of quiescence and development (Fig. 5 and Supplementary Tables 21, 25, 26 and Supplementary Figs. 16, 17). Of the genes with high $F_{\mathrm{ST}}$ values between male and hermaphroditic plants, we focused on the $L E A$ protein gene family, TsF-box genes, and TsSUT genes. Six TsLEA genes (TsLEA2, TsLEA4, TsLEA10, TsLEA12, TsLEA13, and TsLEA19), three F-box genes (TsF-box5, TsF-box9, and TsF-box14), and a TsSUT4 gene were expressed at lower levels during fruit quiescence than at later times when fruits were actively developing (Fig. 5c, d). We found that the glycine-rich protein (GRP) TsGRP1.8 gene was expressed at significantly higher levels during fruit 


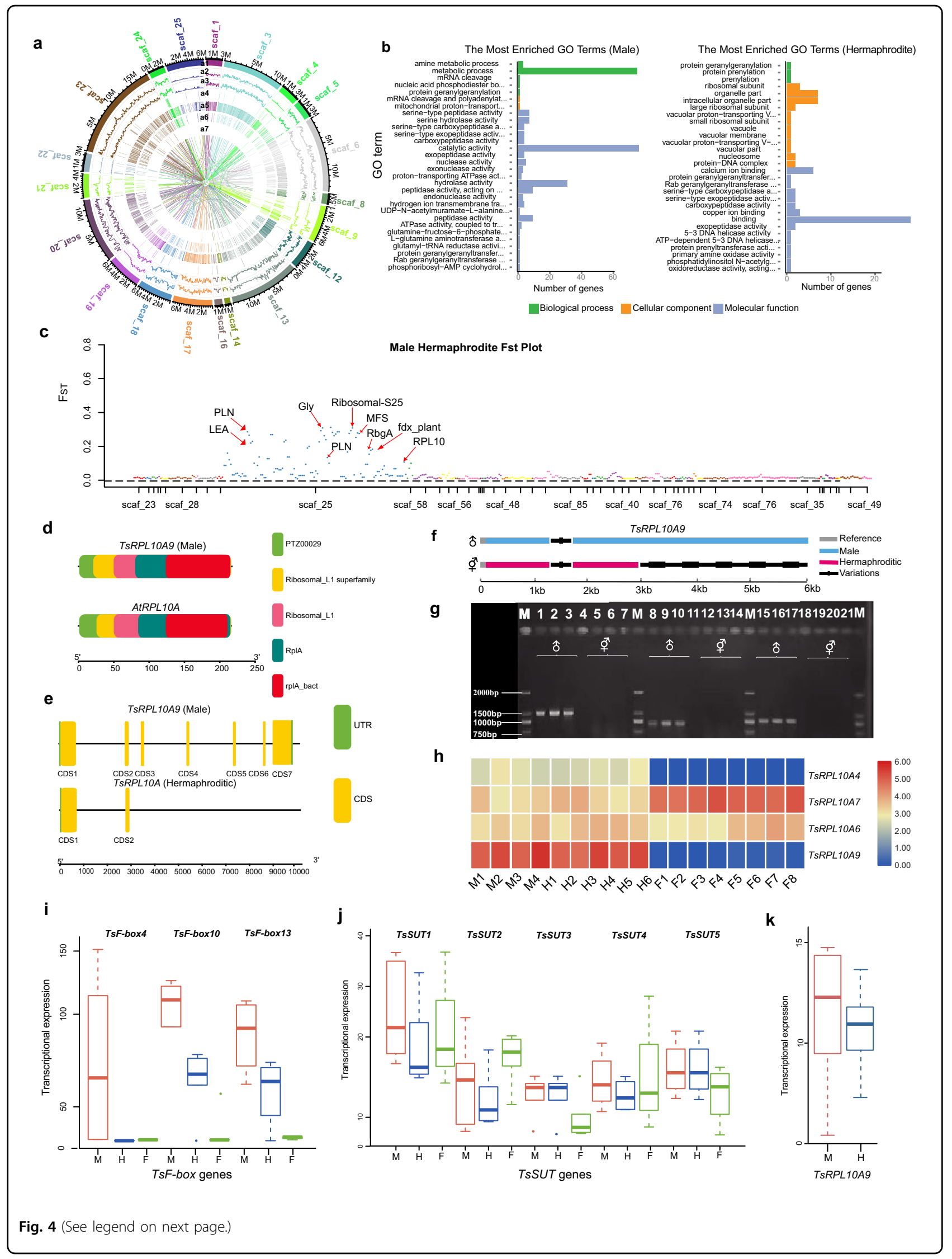


(see figure on previous page)

Fig. 4 Candidate genes associated with sex-linked genomic regions of $\boldsymbol{T}$. sinensis. a Alignment of genomic regions differentially enriched in male versus hermaphroditic groups of T. sinensis. Concentric rings, from outermost to innermost, include "a1"-Scaffolds, "a2"-SNPs, "a3"-Indels, "a4"CNV duplications, "a5"-CNV deletions, "a6"-SV deletions, and "a7"-SV inventions. b The GO enrichments of genes existed only in males and hermaphrodites. c A Manhattan plot showing signatures of sequence divergence between male and hermaphroditic T. sinensis; histograms along each scaffold were based on a $20 \mathrm{~kb}$ window. The dots of different colors indicate regions of selective sweeps associated with sex linkage. Red arrows identify candidate genes with high and significant $F_{\mathrm{ST}}$ values between the genomes of pools of male and hermaphroditic $T$. sinensis. $\mathbf{d}$ Comparison of the RPL10A motif composition of A. thaliana and T. sinensis. e Gene structure of the RPL10A gene in male and hermaphroditic genotypes of T. sinensis compared with A. thaliana. f Molecular structure of TSRPL1OA-M (male) and TSRPL1OA-H (hermaphrodite) alleles verified by Sanger sequencing [the locations of primers (see Supplementary Table 22) are indicated by a light blue line (male) and light red line (hermaphroditic)]. The black line indicates variation (missing sequences) in hermaphroditic T. sinensis. $\mathbf{g}$ The PCR-based products of three male individuals and three hermaphroditic individuals using Sanger sequencing data. Three deletions ( $978 \mathrm{bp}, 659 \mathrm{bp}$, and $773 \mathrm{bp}$ ) within RPL10A were found in hermaphroditic individuals (lanes 4 to 6,11 to 13 , and 19 to 21) compared with male individuals (lanes 1 to 3,8 to 10, and 15 to 17). Lanes 4, 11, and 18 were controls (purified water was used as the template). $\mathbf{h}$ Tissue-specific expression of RPL1OA genes identified in male flowers (M), hermaphroditic flowers (H), and fruits (F) of T. sinensis during the 8 months immediately following fertilization (for details, see Supplementary Table 23 and Supplementary Fig. 13). RPL10A genes and their expression values across different tissues are visualized as a heatmap. Genes associated with differences between the genomes of male and hermaphroditic plants are shown as bars (details on the sampled tissues are provided in Supplementary Table 23). $\mathbf{i}$ The mean expression of three TsF-box (TsF-box4, TsF-box10, and TsF-box13) genes identified in four male flowers (M), six hermaphroditic flowers (H), and eight fruits (F) of T. sinensis (for details on the sampled tissues, see Supplementary Table 24 and Supplementary Fig. 15). $\mathbf{j}$ The mean expression of each of five TSSUT (TSSUT1, TSSUT2, TSSUT3, and TSSUT5) genes in male flowers, hermaphroditic flowers, and fruits of T. sinensis (for details, see Supplementary Table 24 and Supplementary Fig. 14). $\mathbf{k}$ The mean expression of the TSRPL10A9 gene in male flowers (M) versus hermaphroditic flowers (H) of T. sinensis (for details, see Supplementary Table 23 and Supplementary Fig. 13)

quiescence compared with stages of active development (Fig. 5b, d and Supplementary Figs. 16, 17).

\section{Discussion}

\section{Evolution of the androdioecious tree $T$. sinensis}

The genome of the androdioecious tree $T$. sinensis was assembled and evaluated using $154 \mathrm{~Gb}$ of clean read data (343X). The final estimated genome size was $410 \mathrm{Mb}$, among the smallest reported for any tree. The genome was preliminarily annotated using RNA-seq data, from which we identified 22,251 protein-coding genes. Our study included a nearly complete representation and localization of genes, repeat elements, WGD, and RNAs; functional and metabolic annotations; and an analysis of the expression of genes with a putative role in sex determination and fruit development. A comparison of the genome of $T$. sinensis with the genomes of 17 angiosperm plants revealed differences in evolutionary expansion and contraction of gene families, in addition to unique gene families. These data represent an important resource for the study of evolutionary processes, gene evolution and function, molecular genetics and biochemistry in Tapiscia and the Huertales.

Currently, the genus Tapiscia comprises a single species, T. sinensis ${ }^{1-5,24}$, an endangered (Red List) species found in scattered, disjunct populations in subtropical and southwestern central China ${ }^{25-27}$. The T. sinensis habitats in the mountains of subtropical China were fragmented by past climatic changes ${ }^{25}$. Our results based on wholegenome resequencing showed that extant populations have low genetic diversity, are genetically distinct, and show little evidence of genetic exchange among populations (Fig. 2). The same conclusions were reached by
Zhang et al. ${ }^{25}$ based on chloroplast haplotypes. It is possible that fragmentation of $T$. sinensis populations has resulted in populations with unique adaptations (Fig. 2). We suggest that populations of T. sinensis in the Tianmu Mountains, the Qinling Mountains, the Shennong Mountains, the Naling Mountains, and Yunnan Province be given the highest priority for conservation (Fig. 2).

\section{The transcriptomic and developmental bases of androdioecy}

Androdioecy is a rare breeding system (occurring in $<0.005 \%$ of angiosperms) in which populations consist of males and hermaphrodites ${ }^{10}$. The genetic and genomic bases of sex determination in plants are a critical area of study in biology and evolution. Although sex-linked systems are important in some horticultural plant species, such as red bayberry ${ }^{29}$, melons ${ }^{30,31}$, cucurbit ${ }^{32}$, kiwifruit ${ }^{33}$, and persimmons $(O G I)^{34}$, the genomic and genetic bases of androdioecy have not been explored. We identified genomic regions and genes potentially associated with androdioecy by comparing male versus hermaphroditic genome pools. We identified a genomic scaffold (scaffold 25) that was unusually rich in candidate genes (Fig. 4 and Supplementary Tables 18-21). A 24-kb region of scaffold 25 was absent in hermaphroditic individuals. To confirm the function of this $24-\mathrm{kb}$ region and determine its role in the specification of floral sexual development, a more complete, higher-quality genome assembly will be required $^{35-38}$. We showed that the structure and sequence of RPL10A differed strongly between male and hermaphroditic trees, consistent with studies showing that AtRPL10A mutations caused female gametophyte lethality in Arabidopsis ${ }^{39,40}$, and that there were highly significant 


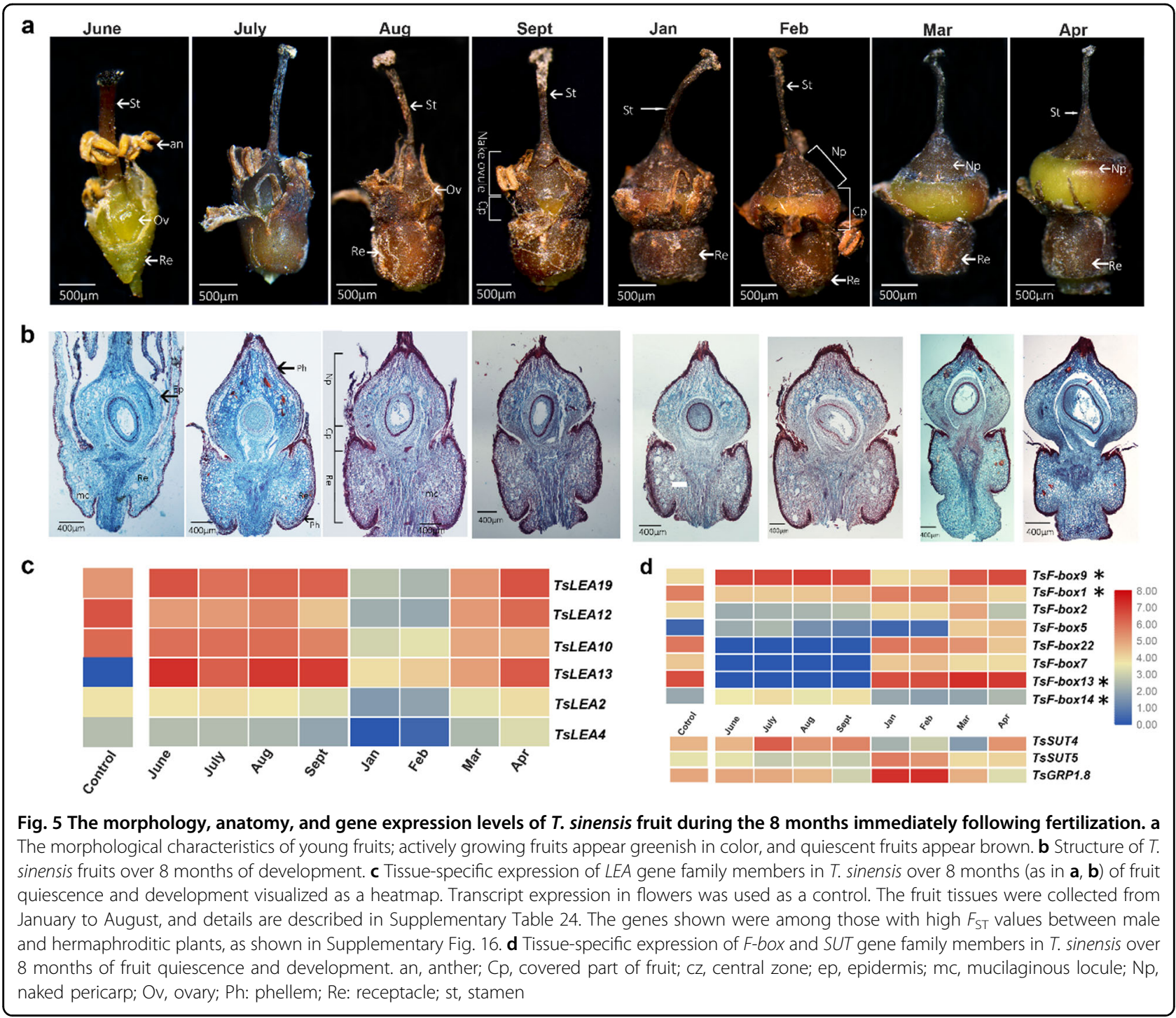

differences in the expression patterns of candidate $T s F$ box and TsSUT genes in male versus hermaphroditic flowers and fruit (Fig. 4).

In previous studies, our results showed that bisexual flowers are the ancestral condition in T. sinensis ${ }^{4-6}$. Loss of female function may be the consequence of a large-scale insertion into TsRPL1OA that caused the development of a sterile pistil in the hermaphroditic flower primordium (Figs. 3 and 4$)^{39}$. T. sinensis, as an androdioecious tree, may maintain characteristics associated with selfincompatibility ${ }^{41}$. We identified self-incompatibilityrelated $F$-box genes in the $T$. sinensis genome, including TsF-box14, TsF-box19, and TsF-box22, which contained a conserved self-incompatibility (S-locus) domain ${ }^{42}$ whose function might be confirmed by transformation into a model such as $A$. thaliana or Nicotiana tabacum ${ }^{43,44}$.

Tapiscia sinensis, although mostly subtropical in its distribution, also occupies habitats where temperatures can fall as low as $-20^{\circ} \mathrm{C}$. Cold hardiness evolves through long-term adaptation to low-temperature environments ${ }^{45-49}$. T. sinensis flowers are fertilized in early June, the young fruits become quiescent in September, and this status is maintained until the following April. Quiescent young fruits have several characteristics that appear to be adaptations to low temperatures, including phellem formation on the ovary surface, an ovary enclosed in a receptacle, zygote quiescence, and protein, carbohydrate and lipid accumulation in the "overwintering complex"1,50. These developmental traits, along with androdioecy, may be adaptations to long-term climatic oscillations ${ }^{1-5,24}$. Among the genes that differed most between male and bisexual flowers, several had potential roles in cellular protection under cold stress or increasing storage proteins ${ }^{51}$. The expression levels of $F$-box genes (TsF-box1, TsF-box22, and TsF-box13) and SUT genes (e.g., TsSUT5) were higher at times, consistent with a role 
in fruit development and quiescence ${ }^{52-54}$ (Fig. 5 and Supplementary Figs. 14, 15). We further showed that the TsGRP1.8 gene was highly expressed during fruit quiescence (Fig. 5d). The GRP1.8 gene is part of a repair system during the stretching phase of protoxylem development ${ }^{53}$ and insolubilized within the cell wall later in development $^{54}$ (Fig. 5 and Supplementary Fig. 17). It is possible that TsGRP1.8 plays a role in increasing the cell wall thickness or fruit stability to survive cold temperatures during quiescence (Fig. 5b, d) ${ }^{55}$.

The pollen of bisexual flowers of $T$. sinensis is round and unlike that of male flowers, a phenomenon also observed in Tetracera oblongata, which is functionally dioecious ${ }^{56}$. This may indicate that pollen of hermaphroditic plants of $T$. sinensis is nonfunctional, a possibility suggested by Charlesworth (1984) as likely based on theoretical considerations ${ }^{57}$. Charlesworth ${ }^{58}$ also considered the 1:1 ratio of male to fruitful plants that we observed to be evidence that hermaphroditic plants were male sterile. We did not observe whether hermaphrodite pollen was fertile but did observe that hermaphrodite pollen can be shed onto adjacent stigmas, an event that would lead to inbreeding unless there is a selfincompatibility system, which we did see evidence of in nine F-box genes (Supplementary Fig. 15). Charlesworth agreed with Sobrevila and Arroyo (1982), who speculated that in Matayaba tovarensis, dioecy evolved from selfincompatibility in hermaphrodites ${ }^{59}$. Charlesworth (1984) further stated that "when pollen is actually produced in females, but is nonfunctional, a role for inbreeding avoidance seems particularly clear", as sterile pollen can still attract pollinators. We did not record whether $T$. sinensis produces nectar as a reward, but TsSUT3 was expressed in flowers but not fruit, so it could be related to nectar production (Supplementary Fig. 14 and Fig. 4i). We also observed that the expression of some SUT genes was consistent with a role in fruit development, which of course only happens in hermaphroditic plants. It makes sense that sucrose transporters would be needed for fruit development.

In conclusion, the genome assembly of $T$. sinensis provides a reference for future genetic, genomic and evolutionary studies in Tapiscia and its relatives. $T$. sinensis was fragmented into five lineages based on wholegenome resequencing data from 10 populations and 55 individuals. A $24-\mathrm{kb}$ region located on scaffold 25 was absent in the hermaphroditic individuals. It was rich in candidate sex-linked genes, e.g., TsRPL1OA $9^{52}$. We further identified eight F-box genes, six LEA genes, four SUT genes, and one GRP gene that are involved in sugar metabolism in fruit, overwintering fruit development, and flower development ${ }^{42-44,53-62}$, potentially indicating that these genes play a role in regulating flower development and fruit quiescence during winter in $T$. sinensis. These genes may be of use for $T$. sinensis breeding or for further identification of genomic regions affecting sex determination. Finally, the identification of sex-linked genes in $T$. sinensis will inform studies on the evolution of androdioecy, sex-linked gene functional analysis, plant sexual development, and fruit development.

\section{Methods \\ Tree samples}

In 2016, we collected leaf samples from a single individual of the androdioecious tree Tapiscia sinensis (wild male individual) growing in the Qinling Mountains,

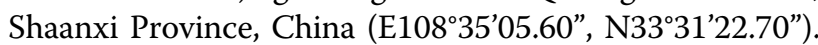
High-quality genomic DNA was extracted from fresh leaf tissues using the Qiagen DNeasy Plant Mini Kit (Qiagen, Dusseldorf, Germany). In 2016, shoots were collected from 55 trees representing 10 wild populations of Tapiscia sinensis in seven Chinese provinces (Supplementary Table 18). These 10 populations represented most of the geographic distribution of this endangered, woody tree species (Fig. 2).

\section{Transcriptome sample preparation and sequencing}

Five tissues (fruits, leaves, stems, roots, and male flowers) were collected from a single male tree of $T$. sinensis (located in the Qinling Mountains, Shaanxi Province, China). We also collected hermaphroditic flowers from a hermaphroditic tree growing in the Qinling Mountains (E $\left.108^{\circ} 35^{\prime}, \mathrm{N} 33^{\circ} 31^{\prime}\right)$. RNA was extracted from these materials with TRI reagent. The RNA-seq data of six tissues were used to make libraries using the TruSeq ${ }^{\circledR}$ RNA Sample Preparation protocol for the Illumina HiSeq 4000 platform. Transcriptomes of 18 independent samples of tissues (four male flowers, six hermaphroditic flowers, and eight fruits) were evaluated for levels of expression using genes identified in T. sinensis (Supplementary Table 24).

\section{Library preparation}

For Illumina sequencing (HiSeq 4000 platform), we used a paired-end library with an insert size of $350 \mathrm{bp}$. Paired-end 10X Genomics genome libraries with insert sizes of $50-100 \mathrm{~kb}$ were constructed using the Illumina NovaSeq platform. Long reads of $20 \mathrm{~kb}$ DNA inserts were constructed for PacBio single-molecule real-time sequencing to assist in genome assembly.

\section{Genome de novo assembly}

Before sequence assembly, "daligner" was executed by the main script of the FALCON assembler to correct errors in the PacBio long reads and to generate consensus sequences $^{63}$. After error correction, the consensus sequences achieved accuracies up to $99.999 \%$. Then, FALCON identified the overlaps between all pairs of the preassembled error-corrected reads. The read overlaps 
were used to construct a directed string graph following Myers' algorithm ${ }^{64}$. Contigs were constructed by finding the paths from the string graph (falcon_sense_option $=--$ output_multi --min_idt 0.70 --min_cov 3 --max_n_read $200--n \_c o r e ~ 10$; overlap_filtering_setting $=--\max _{-}-$ diff 100 --max_cov 100 --min_cov 3 --n_core 24$)^{64}$. Error correction of the preceding assembly was performed using the PacBio "arrow" consensus-calling algorithm ${ }^{65}$. Illumina reads were error-corrected with Pilon ${ }^{66}$. FragScaff software was used to extend 10X Genomics scaffolds as follows: Linked reads generated using the 10X Genomics library were aligned to the consensus sequence of the PacBio assembly to obtain superscaffolds using BOWTIE $\mathrm{v} 2.2^{67}$. As the length of the consensus sequence increases, fewer linked reads supporting connections are required. The consensus sequence with linked-read support was used for the subsequent assembly. The FragScaff parameters were "-fs1 '-m 3000 -q 30 -E 30000 -o 60000' -fs2 'C 5 ' -fs3 ' - j 1 -u $3^{\prime \prime \prime 68}$. We also evaluated the completeness and accuracy of the genome assembly using Bench Marking Universal Single-copy Orthologs (BUSCO) version $4.0 .5^{69}$.

\section{Repeat annotation}

We identified the repeat sequences in the $T$. sinensis genome using LTR_FINDER ${ }^{70}$, PILER-DF ${ }^{71}$, RepeatScout $^{72}$, and MITE-Hunter ${ }^{73}$. The Repbase database ${ }^{74}$ was combined to construct a nonredundant repeat sequence library of the T. sinensis genome. Tandem Repeats Finder was used to identify tandem repeat sequences in the $T$. sinensis genome ${ }^{75}$. MISA software was used to identify the simple sequence repeats (SSRs), with thresholds of ten repeat units for mononucleotide SSRs and five repeat units for di-, tri-, tetra-, penta-, and hexanucleotide SSRs.

\section{Gene prediction and annotation}

To predict genes in the T. sinensis genome, we used two methods: homology-based prediction with BLAST (Evalue $\left.\leq 1 \mathrm{e}^{-5}\right)^{76}$ and GeneWise (version 2.4.1) ) $^{77}$ and $\mathrm{ab}$ initio prediction with Augustus ${ }^{78}$ and GlimmerHMM ${ }^{79}$. We predicted the gene structure of the corresponding genomic regions using BLAST hits through GeneWise (version 2.4.1) ${ }^{77}$. Protein sequences were downloaded from the NCBI to perform homology predictions. RNAseq data derived from six tissues (fruit, leaf, stem, root, male flower, and hermaphroditic flower) were assembled using Trinity. We generated gene models using Evidence Modeler $(\mathrm{EVM})^{80}$. UTR and alternative splicing variation were predicted from gene models using PASA2 software ${ }^{81}$.

We performed functional annotation using BLASTP with an E-value cutoff of $1 \mathrm{e}^{-5}$. Protein-coding genes were identified with searches against SwissProt and TrEMBL ${ }^{82}$. We annotated conserved protein domains using the
InterPro (version 5.16) ${ }^{83}$ and Pfam (v 3.0) databases ${ }^{84}$. We predicted the biological pathways in the KEGG database ${ }^{85}$ using BLAST with an E-value cut-off of $1 \mathrm{e}^{-05}$.

\section{Comparative analysis of $T$. sinensis genome evolution}

We performed global gene family classification of 17 plant genomes, including those of M. notabilis, Z. jujuba, P. persica, O. sativa, A. thaliana, J. regia, J. curcas, G. max, G. raimondii, T. cacao, F. schinensis, P. trichocarpa, $V$. vinifera, C. papaya, S. lycopersicum, O. europaea, and C. sinensis, based on whole protein-coding gene repertoires. We performed gene pair searches within these 17 plant genomes using BLASTP. We clustered gene families from these plant species using OrthoMCL ${ }^{86}$ with the parameter "-inflation 1.5".

\section{Phylogenetic tree reconstruction}

We first generated a multiple sequence alignment with default parameters through MUSCLE ${ }^{84}$. We used ProtTest $^{87}$ to select the best substitution models, and the JTT $+\mathrm{I}+\mathrm{G}+\mathrm{F}$ model was selected as the best-fitting model. Then, we constructed a maximum likelihood (ML) phylogenetic tree based on 509 families' genes using RAxML $^{88}$.

\section{Species divergence time estimation}

We estimated the divergence times for 18 species using MCMCTree in PAML ${ }^{89}$ with the "correlated molecular clock" and "JC69" models. We applied a total of five fossil calibration points for the estimation of divergence times: O. sativa and S. lycopersicum divergence time (115-308 million years ago), $V$. vinifera and $G$. max divergence time (107-135 million years ago), $V$. vinifera and A. thaliana divergence time (107-135 million years ago), J. regia and $P$. trichocarpa divergence time (101-131 million years ago), and $P$. trichocarpa and $A$. thaliana divergence time (98-117 million years ago) (http://www.timetree.org/) (Fig. 1).

\section{Gene family expansion and contraction}

CAFÉ 2.2 $2^{90}$ (Computational Analysis of gene Family Evolution) software was used to assess the expansion and contraction of orthologous gene families among the 18 plant genomes based on a phylogenetic tree.

\section{Whole-genome duplication analysis}

We identified syntenic blocks in the T. sinensis genome using MCScanX with a BLASTP E-value $<1 \mathrm{e}^{-7}$. We calculated the $4 \mathrm{DTv}$ distance for each gene pair in a syntenic block to identify putative whole-genome duplication events in $T$. sinensis. The synonymous substitution rate (Ks) was calculated using the YN model in KaKs_Calculator v2.091. 


\section{Transcriptome library and gene expression analysis}

Transcriptomes $(N=18)$ were mapped to the $T$. sinensis genome using TopHat ${ }^{92}$. The expression of each gene was normalized by read counts (RPKM). We used DESeq to identify differentially expressed genes (Supplementary Table 26). We generated the expression profiles of all differentially expressed genes using gCLUTO (http:// glaros.dtc.umn.edu/gkhome/cluto/gcluto/overview).

\section{Alignment and variation calling from whole-genome resequencing data of 55 accessions}

We mapped the short reads to the $T$. sinensis genome using BWA with the following parameters: bwa mem - $t 4$ $-M-R^{93}$. SAM/BAM files were evaluated to remove PCR duplicates using the Picard package with default parameters (http://broadinstitute.github.io/picard). We computed the coverage and depth of sequence alignments using the DepthOfCoverage program in the Genome Analysis Toolkit ${ }^{94}$ (GATK) and BEDtools ${ }^{95}$, respectively (Supplementary Table 19). We called SNPs following GATK best practices. Low-quality alignments with a mapping quality $<20$ were filtered using SAMtools ${ }^{96}$.

\section{Population genetic analysis}

We constructed a neighbor-joining (NJ) tree of $55 \mathrm{~T}$. sinensis accessions using TreeBeST ${ }^{97}$ and EvolView ${ }^{98}$. We performed PCA using GCTA software with default settings ${ }^{99}$. The top four eigenvectors of samples were plotted using the ggplot2 $\mathrm{R}$ package ${ }^{100}$. We also investigated population structure using ADMIXTURE ${ }^{101}$, wherein the maximum iteration time was set to 10,000 , and the number of population groups $(K)$ was varied from 1 to 10 . We identified sex-linked genomic regions by comparing 26 hermaphroditic individuals and 29 male individuals for regions showing reduction of diversity $\left(\mathrm{ROD}=1-\pi_{\text {male }} /\right.$ $\pi_{\text {hermaphroditic }}$. $F_{\mathrm{ST}}$ parameters were calculated in windows of $10 \mathrm{~kb}$ along the entire $T$. sinensis genome (Supplementary Table 18).

\section{Floral morphology and development}

Living tissues were collected from cultivated trees growing at Northwest University, Shanxi Province (N34 ${ }^{\circ}$ $14^{\prime} 58.61^{\prime \prime}$, E $108^{\circ} 55^{\prime} 39.72$ "). Samples were collected in June, July, August, September, January, February, March, and April. We observed and photographed the morphological features of $T$. sinensis using a stereomicroscope (OLYMPUS SZX12). We prepared paraffin sections using routine methods ${ }^{102}$. Briefly, tissues of $T$. sinensis were dehydrated in an ethanol series (5 min 85\% ETOH, 95\% $\mathrm{ETOH}, 100 \% \mathrm{ETOH}$, and 100\% ETOH), infiltrated with xylene $(20 \mathrm{~min} 1 / 2 \mathrm{ETOH}+1 / 2$ xylene and $15 \mathrm{~min}$ wash in $100 \%$ xylene two times $)^{4}$, and embedded in paraffin for sectioning. The prepared slides were critically observed and photographed using a Nikon DS-Fil digital camera ${ }^{5}$.

\section{Gene families}

Query sequences of RPL10A, SUT, LEA, and F-box genes of $A$. thaliana were downloaded from TAIR (https://www.arabidopsis.org/browse/genefamily/index.

jsp). Gene family size for RPL1OA, SUT, LEA, and F-box gene families was determined using blastp with the default parameter $^{70}$. We characterized the structure of RPL10A, SUT, LEA, and F-box genes using the online software GSDS2.0 $0^{103}$. The protein domains of these four gene families' members were predicted in NCBI software. The $R$ package pheatmap was used to detect the expression pattern of these four gene families (R package) ${ }^{104}$.

\section{PCR verification of RPL10A gene variations}

Total genomic DNA of $T$. sinensis was extracted using the CTAB method ${ }^{4,5}$. We designed six TsRPL10A primer pairs to verify the genetic variation between male and hermaphroditic individuals (Supplementary Table 22). The PCR protocols were as previously reported ${ }^{3-5}$, using the previously mentioned primers (Supplementary Table 22), and the PCR products were verified by agarose gel electrophoresis.

\section{Acknowledgements \\ This study was supported by the National Natural Science Foundation of China (31770413, 31270428, and 41471038). Mention of a trademark, proprietary product, or vendor does not constitute a guarantee or warranty of the product by the U.S. Department of Agriculture and does not imply its approval to the exclusion of other products or vendors that also may be suitable.}

\section{Author contributions}

P.Z. and W.Z.L. conceived the study. W.Z.L., P.Z., X.L.R., H.W., and G.L.X. collected samples and performed experiments. W.Z.L. photographed the morphological features of Tapiscia sinensis. X.L.R., G.L.X., and W.Z.L. analyzed floral morphology and development. W.Z.L., P.Z., and G.L.X. assembled the genome. F.Y., P.Z., G.L. X., X.L.R., and W.Z.L. completed the genome annotation and evolutionary analyses. P.Z. and W.Z.L. wrote the paper. P.Z., K.W., F.Y., and W.Z.L. edited and revised the English writing of this paper. All authors read and approved the final paper.

\section{Data availability}

The raw reads of the genome and the resequencing data for 55 Tapiscia sinensis individuals have been deposited as a BioProject under accession PRJNA587558. The raw transcriptome data for flowers and fruits of $T$. sinensis have been deposited as a BioProject under accession PRJNA284864.

Conflict of interest

The authors declare that they have no conflict of interest.

Supplementary Information accompanies this paper at (https://doi.org/ 10.1038/s41438-020-00414-w).

Received: 11 March 2020 Revised: 14 July 2020 Accepted: 11 August 2020 Published online: 01 December 2020

\footnotetext{
References

1. Liu, W. Z., Kang, H. Q., Zheng, H. C. \& Feng, Y. Z. An investigation on the sexual reproductive cycle in Tapiscia sinensis. J. Syst. Evol. 46, 175-182 (2008).

2. Zhou, X. J., Ma, L. \& Liu, W. Z. Functional androdioecy in the rare endemic tree Tapiscia sinensis. Bot. J. Linn. Soc. 180, 504-514 (2016).
} 
3. Yang, $K$. et al. Carbohydrate metabolism and gene regulation during anther development in an androdioecious tree, Tapiscia sinensis. Ann. Bot. $\mathbf{1 2 0}$ 967-977 (2017).

4. Xin, G. L. et al. Floral development in the androdioecious tree Tapiscia sinensis: implications for the evolution to androdioecy. J. Syst. Evol. https://doi. org/10.1111/jse.12517 (2019).

5. Ren, X. L. et al. Abnormal 1 tapetum development in hermaphrodites of an androdioecious tree, Tapiscia sinensis. Tree Physiol. 40, 108-118 (2019).

6. Xin, G. L. et al. Anatomy and RNA-Seq reveal important gene pathways regulating sex differentiation in a functionally Androdioecious tree, Tapiscia sinensis. BMC Plant Biol. 19, 554 (2019).

7. Pannell, J. R. The evolution and maintenance of androdioecy. Annu. Rev. Ecol. Evol. S. 33, 397-425 (2002)

8. Choudhury, B. I., Khan, M. L. \& Dayanandan, S. Functional androdioecy in critically endangered Gymnocladus assamicus (Leguminosae) in the eastern Himalayan region of northeast India. PLOS ONE 9, e87287 (2014).

9. Akimoto, J., Fukuhara, T. \& Kikuzawa, K. Sex ratios and genetic variation in a functionally androdioecious species, Schizopepon bryoniaefolius (Cucurbitaceae). Am. J. Bot. 86, 880-886 (1999).

10. Vernet, $\mathrm{P}$. et al. Evidence for the long-term maintenance of a rare selfincompatibility system in Oleaceae. N. Phytol. 210, 1408-1417 (2016).

11. Liston, A., Rieseberg, L. H. \& Elias, T. S. Functional androdioecy in the flowering plant Datisca glomerata. Nature 343, 641-642 (1990).

12. Wolf, D. E., Satkoski, J. A., White, K. \& Rieseberg, L. H. Sex determination in the androdioecious plant Datisca glomerata and its dioecious sister species D. cannabina. Genetics 159, 1243-1257 (2001).

13. Landry, C. L. \& Beverly, J. R. Do inbreeding depression and relative male fitness explain the maintenance of androdioecy in white mangrove, Laguncularia racemosa (Combretaceae)? N. Phytol. 176, 891-901 (2007)

14. $\mathrm{Xu}, \mathrm{Y}$. C. et al. The differentiation and development of pistils of hermaphrodites and pistillodes of males in androdioecious Osmanthus fragrans $\mathrm{L}$. and implications for the evolution to androdioecy. Plant Syst. Evol. 300, 843-849 (2014).

15. Duan, Y. et al. Assessment of genetic diversity among androdioecious ancient Osmanthus fragrans trees by SSR markers. Plant Syst. Ecol. 61, 179-185 (2015).

16. APG II. An update of the Angiosperm Phylogeny Group classification for the orders and families of flowering plants: APG II. Bot. J. Linn. Soc. 141, 399-436 (2003).

17. Tang, Z. X. et al. Investigation on sexual flowering and fruiting periods in Torreya garandis. J. Syst. Evol. 24, 447-453 (1986).

18. Chen, Z. D., Xing, S. P., Liang, H. X. \& Lu, A. M. Morphogenesis of female reproductive organs in Carpinus turczaninowii and Ostryopsis davidiana (Betulaceae). Acta Bot. Sin. 43, 1110-1114 (2001).

19. Liu, X. \& Yang, C. Temporal characteristics of developmental cycles of female and male flowers in Betula platyphylla in northeastern China. Sci. Silvae Sin. 42, 28-42 (2006)

20. Zhang, P. et al. Development and characterization of 11 polymorphic microsatellite markers in Tapiscia sinensis (Staphyleaceae). Appl. Plant Sci. 1 1300051 (2013).

21. Rüffle, L. et al. Myricaceae, Leguminosae, Icacinaceae, Sterculiaceae, Nymphaeaceae, Monocotyledones, Coniferae. Eozäne Floren des Geiseltales. Abh. Zentr. Geol. Inst. Paläont. Abh. 26, 199-238 (1976).

22. Manchester, S. R. Fruits and seeds of Tapiscia (Staphyleaceae) from the middle Eocene of Oregon. Usa. Tert. Res. 9, 59-66 (1987).

23. Chandrasekharam, A. Megafossil flora from the Genesee locality, Alberta, Canada. Palaeontogr. Abt. B. 147, 1-41 (1974).

24. Eppley, S. M. \& John, R. P. Density-dependent self-fertilization and male versus hermaphrodite siring success in an androdioecious plant. Evolution 61, 2349-2359 (2007).

25. Zhang, J. et al. Phylogeography and genetic structure of a tertiary relict tree species, Tapiscia sinensis (Tapisciaceae): implications for conservation. Ann. Bot. 116, 727-737 (2015).

26. Xie, C. P. A Review of research advances in rare and endangered plant Tapiscia sinensis. Subtropical Plant Sci. 35, 71-74 (2006).

27. Ren, X. L. et al. Characterization of the complete chloroplast genome sequence of Tapiscia sinensis (Tapisciaceae). Conserv. Genet. Resour. 10, 765-768 (2018).

28. Zhou, X. J. et al. De novo characterization of flower bud transcriptomes and the development of EST-SSR markers for the endangered tree Tapiscia sinensis. Int. J. Mol. Sci. 16, 12855-12870 (2015).
29. Jia, H. M. et al. The red bayberry genome and genetic basis of sex determination. Plant. Biotechnol. J. 17, 397-409 (2019)

30. Boualem, A. et al. A conserved mutation in an ethylene biosynthesis enzyme leads to andromonoecy in melons. Science 321, 836-838 (2008).

31. Martin, A. et al. A transposon-induced epigenetic change leads to sex determination in melon. Nature 461, 1135-1138 (2009).

32. Boualem, A. et al. A cucurbit androecy gene reveals how unisexual flowers develop and dioecy emerges. Science 350, 688-691 (2015).

33. Akagi, T. et al. A Y-encoded suppressor of feminization arose via lineagespecific duplication of a cytokinin response regulator in kiwifruit. Plant Cell 30, 780-795 (2018)

34. Akagi, T., Henry, I. M., Tao, R. \& Comai, L. A Y-chromosome-encoded small RNA acts as a sex determinant in persimmons. Science 346, 646-650 (2014).

35. Massonnet, M. et al. The genetic basis of sex determination in grapes. Nat. Commun. 11, 2902 (2020).

36. Müller, N. A. et al. A single gene underlies the dynamic evolution of poplar sex determination. Nat. Plants 6, 630-637 (2020).

37. Li, W. et al. Fine mapping of the sex locus in Salix triandra confirms a consistent sex determination mechanism in genus Salix. Hortic. Res. 7, 64 (2020).

38. Wang, Y. et al. Construction of an anchoring SSR marker genetic linkage map and detection of a sex-linked region in two dioecious populations of red bayberry. Hortic. Res. 7, 53 (2020).

39. Imai, A., Komura, M., Kawano, E., Kuwashiro, Y. \& Takahashi, T. A semidominant mutation in the ribosomal protein L10 gene suppresses the dwarf phenotype of the acl5 mutant in Arabidopsis thaliana. Plant J. 56, 881-890 (2008)

40. María, L. F. F., Alejandro, P., Jordane, B., Alma, L. B. \& Paula, C. Plant L10 ribosomal proteins have different roles during development and translation under ultraviolet-B stress. Plant Physiol. 153, 1878-1894 (2010).

41. Appanah, S. Pollination of androdioecious Xerospermum intermedium Radlk. (Sapindaceae) in a rain forest. Biol. J. Linn. Soc. 18, 11-34 (1982).

42. Lai, Z. et al. An F-box gene linked to the self-incompatibility (S) locus of Antirrhinum is expressed specifically in pollen and tapetum. Plant Mol. Biol. 50, 29-42 (2002).

43. Nasrallah, M. E., Pei, L. \& June, B. N. Generation of self-incompatible Arabidopsis thaliana by transfer of two S locus genes from A. lyrata. Science $\mathbf{2 9 7}$ 247-249 (2002).

44. Jane, M. et al. Expression of a Self-Incompatibility Glycoprotein (S2-Ribonuclease) from Nicotiana alata in Transgenic Nicotiana tabacum. Plant Cell 4, 1063-1074 (1992).

45. Xu, C. X. Research progress on the mechanism of improving plant cold hardiness. Acta Ecol. 32, 7966-7980 (2012).

46. Cao, Q., Kong, W. \& Wen, P. Plant freezing tolerance and genes express in cold acclimation: a review. Acta Ecol. Sin. 24, 806-811 (2004).

47. Kalberer, S. R., Wisniewski, M. \& Arora, R. Deacclimation and reacclimation of col d-hardy plants: current understanding and emerging concepts. Plant Sci. 171, 3-16 (2006).

48. Charrier, G., Ngao, J., Saudreau, M. \& Améglio, T. Effects of environmental factors and management practices on microclimate, winter physiology, and frost resistance in trees. Front. Plant Sci. 6, 259 (2015).

49. Guàrdia, M. et al. Genetics of frost hardiness in Juglans regia L. and relationship with growth and phenology. Tree Genet. Genomes 12, 83 (2016).

50. Teng, L. \& Liu, W. Z. Passing winter strategies of Tapiscia sinensis during its extreme long reproductive cycle. J. Wuhan. Bot. Res 27, 70-75 (2009).

51. Huang, Y. J. et al. The genomes of pecan and Chinese hickory provide insights into Carya evolution and nut nutrition. GigaScience 8, giz036 (2019).

52. Ferreyra, M. L. F., Casadevall, R., Luciani, M. D., Pezza, A. \& Casati, P. New evidence for differential roles of 110 ribosomal proteins from Arabidopsis. Plant Physiol. 163, 378-391 (2013).

53. Ringli, C., Keller, B. \& Ryser, U. Glycine-rich proteins as structural components of plant cell walls. Cell Mol. Life Sci. 58, 1430-1441 (2001)

54. Condit, C. M. \& Richard, B. M. A gene encoding a novel glycine-rich structural protein of petunia. Nature 323, 178 (1986).

55. Ringli, C., Keller, B. \& Ryser, U. Glycine-rich proteins as structural components of plant cell walls. Cell Mol. Life Sci. 58, 1430-1441 (2001).

56. Charlesworth, D. Androdioecy and the evolution of dioecy. Biol. J. Linn. Soc 22, 333-348 (1984)

57. Lloyd, D. G. The maintenance of gynodioecy and androdioecy in angiosperms. Genetica 45, 325-339 (1975). 
58. Symon, D. E. Sex forms in Solanum (Solanaceae) and the role of pollen collecting insects. In Linnean Society symposium series 385-397 (1979).

59. Bovrevila, C. \& Arroyo, M. T. K. Breeding systems in a montane cloud forest in Venezuela. Plant Syst. Evol. 140, 19-37 (1982)

60. Gao, J. \& Ting, L. Functional characterization of the late embryogenesis abundant (LEA) protein gene family from Pinus tabuliformis (Pinaceae) in Escherichia coli. Sci. Rep. 6, 19467 (2016).

61. Ting, L., Gao, J. \& Zeng, Q. Y. Genome-wide analysis of the LEA (late embryogenesis abundant) protein gene family in Populus trichocarpa. Tree Genet. Genomes 9, 253-264 (2013).

62. Bai, Y. Q. \& Yang, Q. C. Study of late embriogenesis abundant protein. Biotechnol. Bull. 9, 1-7 (2009).

63. Chin, C. S. et al. Phased diploid genome assembly with single molecule realtime sequencing. Nat. Methods 13, 1050-1056 (2016).

64. Myers, E. W. The fragment assembly string graph. Bioinformatics 21, 79-85 (2005)

65. Lin, H. H., Liao, Y. C. \& Dutilh, B. E. Evaluation and validation of assembling corrected PacBio long reads for microbial genome completion via hybrid approaches. PLOS ONE 10, e0144305 (2015).

66. Walker, B. J. et al. Pilon: an integrated tool for comprehensive microbial variant detection and genome assembly improvement. PLOS ONE 9, e112963 (2014).

67. Langmead, B. \& Salzberg, S. L. Fast gapped-read alignment with Bowtie 2. Nat. Methods 9, 357-359 (2012)

68. Adey, A. et al. In vitro, long-range sequence information for de novo genome assembly via transposase contiguity. Genome Res 24, 2041-2049 (2014).

69. Simão, F. A., Waterhouse, R. M., loannidis, P., Kriventseva, E. V. \& Zdobnov, E. M. BUSCO: assessing genome assembly and annotation completeness with single-copy orthologs. Bioinformatics 31, 3210-3212 (2015).

70. Xu, Z. \& Wang, H. LTR_FINDER: an efficient tool for the prediction of fulllength LTR retrotransposons. Nucleic Acids Res. 53, W265-W268 (2007).

71. Edgar, R. C. MUSCLE: multiple sequence alignment with high accuracy and high throughput. Nucleic Acids Res. 32, 1792-1797 (2004).

72. Price, A. L., Jones, N. C. \& Pevzner, P. A. 2005. De novo identification of repeat families in large genomes. Bioinformatics 1, 351-358 (2005).

73. Han, Y. \& Wessler, S. R. MITE-Hunter: a program for discovering miniature inverted-repeat transposable elements from genomic sequences. Nucleic Acids Res. 38, e199 (2010)

74. Bao, W. D., Kojima, K. K. \& Kohany, O. Repbase Update, a database of repetitive elements in eukaryotic genomes. Mob. DNA 6, 11 (2015).

75. Benson, G. Tandem repeats finder: a program to analyze DNA sequences. Nucleic Acids Res. 27, 573-580 (1999).

76. Johnson, M. et al. NCBI BLAST: a better web interface. Nucleic Acids Res. 36, W5-W9 (2008)

77. Birney, E., Michele, C. \& Richard, D. GeneWise and genomewise. Genome Res. 14, 988-995 (2004)

78. Stanke, M. et al. AUGUSTUS: ab initio prediction of alternative transcripts. Nucleic Acids Res. 34, 435-439 (2006).

79. Majoros, W. H., Mihaela, P. \& Steven, L. S. TigrScan and GlimmerHMM: two open source ab initio eukaryotic gene-finders. Bioinformatics 20, 2878-2879 (2004)

80. Haas, B. J. et al. Automated eukaryotic gene structure annotation using EVidenceModeler and the program to assemble spliced alignments. Genome Biol. 9, R7 (2008).
81. Rodríguez, F. A. et al. Novel and recurrent COL7A1 mutations in Chilean patients with dystrophic epidermolysis bullosa. J. Dermatol. Sci. 65, 149-152 (2012).

82. Bairoch, A. \& Rolf, A. The SWISS-PROT protein sequence database and its supplement TrEMBL in 2000. Nucleic Acids Res. 28, 45-48 (2000).

83. Mulder, N. \& Apweiler, R. Interpro and InterProScan: tools for protein sequence classification and comparison. Methods Mol. Biol. 396, 59-70 (2007).

84. Bateman, A. et al. The Pfam protein families database. Nucleic Acids Res. 32 D138-D141 (2004)

85. Kanehisa, M. \& Susumu, G. KEGG: kyoto encyclopedia of genes and genomes. Nucleic Acids Res. 28, 27-30 (2000).

86. Li, L., Stoeckert, C. J. \& Roos, D. S. OrthoMCL: identification of ortholog groups for eukaryotic genomes. Genome Res. 13, 2178-2189 (2003).

87. Abascal, F., Zardoya, R. \& Posada, D. ProtTest: selection of best-fit models of protein evolution. Bioinformatics 21, 2104-2105 (2005).

88. Stamatakis, A. RAXML-VI-HPC: maximum likelihood-based phylogenetic analyses with thousands of taxa and mixed models. Bioinformatics 22, 2688-2690 (2006).

89. Yang, Z. PAML 4: phylogenetic analysis by maximum likelihood. Mol. Biol. Evol. 24, 1586-1591 (2007).

90. Bie, T. D., Cristianini, N., Demuth, J. P. \& Hahn, M. W. CAFE: a computational tool for the study of gene family evolution. Bioinformatics 22, 1269-1271 (2006).

91. Wang, D., Zhang, Y., Zhang, Z., Zhu, J. \& Yu, J. KaKs_Calculator 2.0: a toolkit incorporating gamma-series methods and sliding window strategies. Genom. Proteom. Bioinf. 8, 77-80 (2010).

92. Cole, T. \& Salzberg, P. L. S. L. TopHat: discovering splice junctions with RNASeq. Bioinformatics 9, 9 (2009).

93. Li, H. \& Richard, D. Fast and accurate short read alignment with Burrows-Wheeler transform. Bioinformatics 25, 1754-1760 (2009).

94. McKenna, A. et al. The Genome analysis toolkit: a MapReduce framework for analyzing next-generation DNA sequencing data. Genome Res. 20, 1297-1303 (2010).

95. Quinlan, A. R. \& Ira, M. H. BEDTools: a flexible suite of utilities for comparing genomic features. Bioinformatics 26, 841-8426 (2010).

96. $\mathrm{Li}, \mathrm{H}$. et al. The sequence alignment/map format and SAMtools. Bioinformatics 25, 2078-2079 (2009).

97. Vilella, A. J. et al. EnsemblCompara GeneTrees: complete, duplication-aware phylogenetic trees in vertebrates. Genome Res. 19, 327-335 (2009).

98. He, Z. L. et al. Evolview v2: an online visualization and management tool for customized and annotated phylogenetic trees. Nucleic Acids Res. 44 W236-W241 (2016).

99. Yang, J., Lee, S. H., Goddard, M. E. \& Visscher, P. M. GCTA: a tool for genomewide complex trait analysis. Am. J. Hum. Genet. 88, 76-82 (2011).

100. Ginestet, C. ggplot2: elegant graphics for data analysis. J. R. Stat. Soc. 174, 245-246 (2011).

101. Price, A. L. et al. Principal components analysis corrects for stratification in genome-wide association studies. Nat. Genet. 38, 904-909 (2006).

102. Ruzin, S. E. Plant Microtechnique and Microscopy (Oxford University Press, New York) Vol. 198 (1999).

103. Hu, B. et al. GSDS 2.0: an upgraded gene feature visualization server. Bioin formatics 31, 1296-1297 (2014).

104. Kolde, R. pheatmap: Pretty Heatmaps. R package version 1.0.2 ed. (2015). 